\title{
Correction to: Motor impulsivity and delay intolerance are elicited in a dose-dependent manner with a dopaminergic agonist in parkinsonian rats
}

\author{
Haritz Jiménez-Urbieta ${ }^{1}$ - Belén Gago ${ }^{2}$ - Ana Quiroga-Varela ${ }^{3} \cdot$ Tatiana Rodríguez-Chinchilla $^{3}$ • Leyre Merino-Galán ${ }^{3,4}$. \\ Manuel Delgado-Alvarado ${ }^{5,6,7}$. Irene Navalpotro-Gómez ${ }^{8} \cdot$ Arantzazu Belloso-Iguerategui $^{3} \cdot$ Concepció Marin $^{9}$. \\ María C. Rodríguez-Oroz $3,10,11,12$
}

Published online: 3 July 2020

(C) Springer-Verlag GmbH Germany, part of Springer Nature 2020

\section{Correction to: Psychopharmacology}

$$
\text { https://doi.org/10.1007/s00213-020-05544-6 }
$$

In the original version of this article, the Fig. 3 was published in an incorrect format, even though the data and the related information in the text are correct. This error has no effect on any of the results or interpretations and conclusions. Amendment shows the correct figure. The authors would like to apologize for any trouble this error might have caused.

The online version of the original article can be found at https://doi.org/ 10.1007/s00213-020-05544-6

María C. Rodríguez-Oroz

mcroroz@unav.es

1 Instituto de Investigación Sanitaria Biodonostia, San Sebastián, Spain

2 Instituto de Investigación Biomédica de Málaga, Facultad de Medicina, Universidad de Málaga, Málaga, Spain

3 Centro de Investigación Médica Aplicada (CIMA), Universidad de Navarra, Pamplona, Spain

4 University of the Basque Country (UPV/EHU), Leioa, Spain

5 Neurology Department, Sierrallana Hospital, Torrelavega, Spain

6 Psychiatry Research Area, IDIVAL, University HospitalMarqués de Valdecilla, Santander, Spain
Biomedical Research Networking Center for Mental Health (CIBERSAM), Madrid, Spain

8 Institut Hospital del Mar d'Investigacions Mèdiques, Barcelona, Spain

9 IRCE, Institut d' investigacions Biomèdiques August Pi i Sunyer (IDIBAPS), Barcelona, Spain

10 Ikerbasque Foundation, Bilbao, Spain

11 Basque Center on Cognition, Brain and Language (BCBL), San Sebastián, Spain

12 Servicio de Neurología, Clínica Universidad de Navarra, Pamplona, Spain 
Fig. 3 Behavioural measurements in the VDS.

Premature responses (PMR) in the experimental groups at different conditions. Pretreat, pretreatment; Posttreat, posttreatment;

PPX, pramipexole. $* p<0.05$,

$* * * p<0.001$ vs sham+vehicle;

$\& \& p<0.01$ vs sham+PPX;

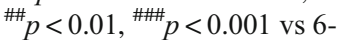

OHDA+vehicle; ${ }^{\text {aaa }} p<0.001$ vs

presurgery; ${ }^{\mathrm{bbb}} p<0.001$ vs pre-

treatment; ${ }^{\mathrm{cc}} p<0.01 \mathrm{vs}$

$0.25 \mathrm{mg} / \mathrm{kg} ;{ }^{\text {ddd }} p<0.001 \mathrm{vs}$

posttreatment
A

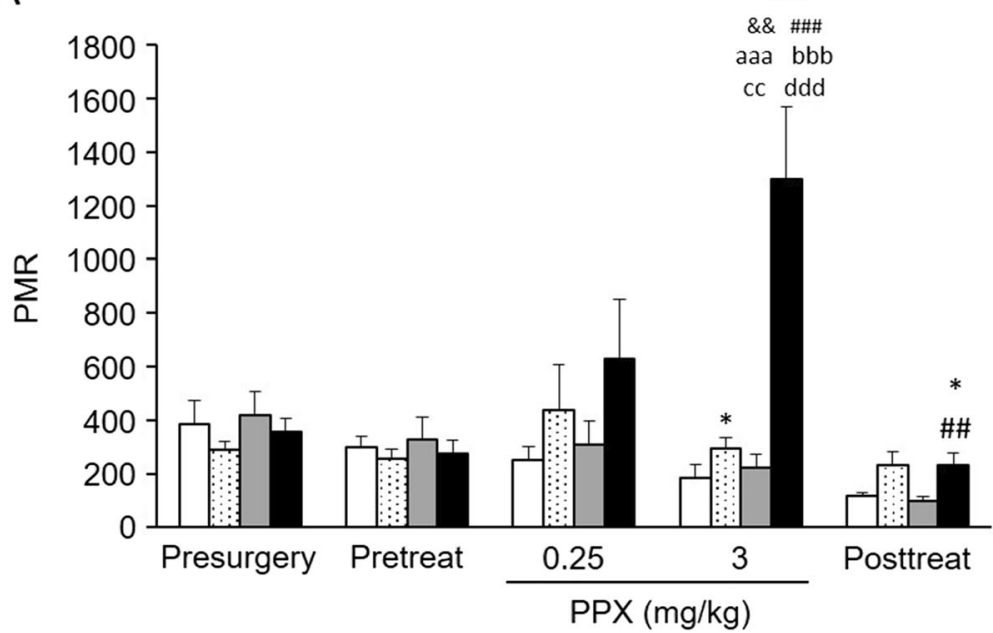

Publisher's note Springer Nature remains neutral with regard to jurisdictional claims in published maps and institutional affiliations. 